\title{
EDITORIAL Gene therapy_from small beginnings to where we are now
}

\author{
Gene Therapy (2017) 24, 495-496; doi:10.1038/gt.2017.23
}

The journal Gene Therapy began 1994 when Joe Glorioso along with Karol Sikora were asked by Nature Publishers to launch a new journal dealing with the rapidly growing science of gene therapy (GT). The goal of the Gene Therapy journal was to host GT research and clinical practice and serve as a bellwether publication for this emerging field. GT got off to a vigorous start and many significant papers have appeared in the journal over the years. Nick Lemoine and Joe Glorioso joined forces 20 years ago to husband the journal with Nick serving as the European Editor and Joe as the American Editor. We have witnessed dramatic ups and downs of the field, where great promise abounded and yet there was frustration with the slowness of development and even a sense of danger. This was highlighted by a lethal inflammatory response to an adenoviral viral vector to treat ornithine decarboxylase deficiency by direct in vivo gene transfer to the liver, and later the induction of leukemia in the treatment of severe combined immunodeficiency following ex vivo gene transfer using retrovirus delivery to subsequently transplanted bone marrow stem cells to correct a gamma chain deficiency. Now there are several gene therapies approved in the US, Europe and China to treat cancer and rare metabolic diseases. GT societies were launched in the US, Europe and Japan followed by many other countries. Multiple gene therapy journals sponsored by interested societies have arisen and many high-profile and more broadly focused journals have actively published gene therapy research. Since this early beginning, the journal has persisted for 22 years and has published 4102 articles not including abstracts, reviews and editorials. Publications in GT have resulted in literature citations amounting to 155,874 to date. While we have decided that the mantle of this journal should be passed on to Rafael J YáñezMuñoz, based at Royal Holloway, University of London, we are confident in the journal's future success and high impact on the field.

Research in the field of GT was largely concerned with the development of gene transfer tools that included engineered viruses and non-viral delivery tools. The concept of altering the genetic make-up of cells and tissues to treat human geneticsbased and acquired disease was exciting and novel, and it seemed like a straightforward direction enabling delivery of new genetic information to functionally defective cells in a safe and directed manner. The natural history of virus infection and persistence in both dividing and non-dividing cells in a variety of species, including humans, made viruses likely candidates to achieve the goals of GT. The reining in of viruses to treat rather than cause disease brought on years of work in different recombinant virus systems to achieve the goals of gene therapy. The trick was to swap pathogenic viral functions with genes that would complement defective cellular activities in the appropriate cell type. Therapeutic gene expression must be durable and persist longterm. There are hundreds of genetic diseases that theoretically could be treated by gene complementation provided that a virus or other type of gene transfer vector could be made available. All of these diseases were rare in incidence with few or no treatment options, and provided targets for proof of concept for GT in which single genes were responsible for the disease phenotype. Most promising was the use of gene transfer to treat recessive diseases where one good gene could solve the problem. Autosomal dominant diseases were more difficult since knockdown or knockout of the defective gene was required before gene addition could be effective.

It seems to us that the GT field has created a large array of gene transfer tools that have enabled an enormous contribution to the development of an in-depth understanding of the pathobiology of disease and continues to lead the way to applications of far more challenging diseases such as cancer, aging, cardiovascular and neurologic disorders. Gene transfer has been complemented most recently by gene editing and potentially gene correction using technologies, such as engineered endonucleases, including CRISPR/Cas, and represents part of the exciting future of GT. It is hard to imagine where biological research would be without the worldwide efforts of gene therapists to introduce genes into cells both ex vivo and in vivo and to think about engineering animal models of human disease, as well as induced pluripotent stem cells to create human disease in a Petri dish. GT has clearly revolutionized the power of biological and disease research in an unprecedented manner.

While there have been many hurdles to overcome that relate to safety, immunity and manufacturing, still, the logic of this treatment modality remains intact and most of us in the field believe that it will take its place as a standard of care just as the use of monoclonal antibodies and pharmaceutical drugs of all types has become widely employed. This is especially true for diseases that are too complex to treat with single approaches, such as cancer.

GT is one of the most highly regulated therapeutic fields. There were numerous control bodies formed to understand the long-term impact of gene therapy and to consider the dangers of viral vector engineering and transfer to humans. The $\mathrm{NIH}$ recombinant DNA subcommittee on gene therapy was specifically charged with review of GT protocols and remains a functioning body; however, the experience with clinical application of some vectors has reduced risk and there is far more insight into the application of vector systems to specific tissues and disease processes.

It takes an enormous amount of effort and stamina to mount a gene therapy clinical trial and exceeds the budgets of most grants and contracts. Yet the promise of creating a therapy for a devastating human disease has driven the biotechnology community to invest huge amounts of capital to realize the potential benefits of gene therapy. GT has also attracted the interest of mainstream pharmaceutical industries who have come on board to bring GT into the market place. While the risk inherent in GT success is quite high, the rewards are potentially enormous both from a commercial as well as human medicine point of view.

We believe that GT and the journal of GT will succeed going forward. There are many fields such as organ transplantation technology that have taken many years to develop before they have become standard practice, and no doubt gene therapy will take a similar path. We predict that by midcentury, there will be many GT options for patients, and in combination with other treatment strategies, will move human medicine to reach heights that are currently unanticipated. The challenge will be how to deliver what are now expensive and difficult treatments to people worldwide who have less developed health care systems and wealth. Our sense is that as we create 
better treatment options, their costs will come down and rival immunization protocols.

\section{CONFLICT OF INTEREST}

The authors declare no conflict of interest.
JC Glorioso ${ }^{1}$ and N Lemoine ${ }^{2}$ ${ }^{1}$ Department of Microbiology and Molecular Genetics, University of Pittsburgh, School of Medicine, Pittsburgh, PA, USA and ${ }^{2}$ Barts Cancer Institute, Queen Mary University of London, London, UK E-mail: glorioso@pitt.edu 